\title{
Diseño y dimensionamiento de plantas solares autónomas para viviendas típicas de la zona rural del Departamento de Córdoba
}

\author{
José Rafael Narvaez Altamiranda ${ }^{1 \star}$, Felix de Jesus Aguas Lastre ${ }^{1 \star \star}$, Juan \\ Manuel Oviedo Cuéter ${ }^{1 \star \star \star}$ \\ Grupo de Materiales y Física Aplicada, Universidad de Córdoba. Montería, Colombia
}

Fecha De Entrega: Marzo 14 DE 2012

Fecha de EVAluación: ABRIL 4 DE 2012

FECha DE APROBACión: MAYO 3 DE 2012

\begin{abstract}
Resumen Se dimensionó un sistema fotovoltaico para que opere en forma autónoma y para que suministre potencia eléctrica a la carga típica de una vivienda en zonas rurales del departamento de Córdoba. En el dimensionamiento de un sistema fotovoltaico uno de los aspectos principales a tener en cuenta es conocer las HSP (horas pico de radiación solar estándar) de la región donde se instala el sistema. Por esta razón se instaló en el municipio de Lorica un sistema de medición de radiación y temperatura adquirida a partir de un sistema de adquisición de datos de la Nacional Instruments y controlada por el software de programación gráfica LabVIEW de la National Instruments [1]. Con los resultados obtenidos de la estación, se dimensión e instaló un sistema fotovoltaico autónomo para una vivienda localizada en la vereda Ceiba Pareja con una potencia nominal de $580 \mathrm{Wp}$.
\end{abstract}

Palabras Clave: sistema fotovoltaico, instrumentación virtual, energía solar, radiación solar, energías alternativas.

Abstract A photovoltaic system was dimensioned in order to operate
autonomously and to supply electrical power to the typical charge of a
house within the rural area of the state of Córdoba. One of the aspects
to keep into account when dimensioning a photovoltaic system is to know
the PSH (standard peak sun hours) of the region where the system is
installed. For this reason, in the municipality of Lorica, we installed a
system to measure the radiation and temperature acquired from a system
to acquire data from National Instruments, and controlled by the graphic
programming software LabVIEW from National Instruments [1]. With
the results obtained from the station, we could dimension and install an
autonomous photovoltaic system for a house located in the township of
Ceiba Pareja with nominal power of $580 \mathrm{Wp.}$

Keywords: photovoltaic system, virtual instruments, solar energy, alternative energies.

\footnotetext{
* jonal73@hotmail.com

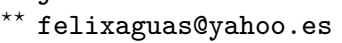

$\star \star \star$ ocjm19@gmail.com
} 


\section{Introducción}

Este trabajo se enmarca en el campo de las energías renovables con el fin de incentivar el uso de éste tipo de tecnologías en el país, y en especial la energía solar fotovoltaica (FV).

Las energías renovables son aquellas que son inagotables, tengan o no su origen en el sol. Las fuentes de energías renovables incluyen la biomasa tradicional, las grandes centrales hidroeléctricas y las nuevas fuentes renovables (solar térmica, solar fotovoltaica, eólica, biomasa moderna, biocombustible, geotérmica y pequeñas centrales hidroeléctricas). Estas contribuyeron para el año 2006 con un $18 \%$ del suministro de energía global primaria mundial, como se muestra en la figura 1 [2].

En la figura 2 se muestra cómo ha sido el crecimiento mundial de las energía renovables entre el 2002 y el 2006 [2], destacándose la generación FV interconectada como la de mayor crecimiento $(60 \%)$ y la generación hidroeléctrica a gran escala como la de menor crecimiento $(2.5 \%)$.

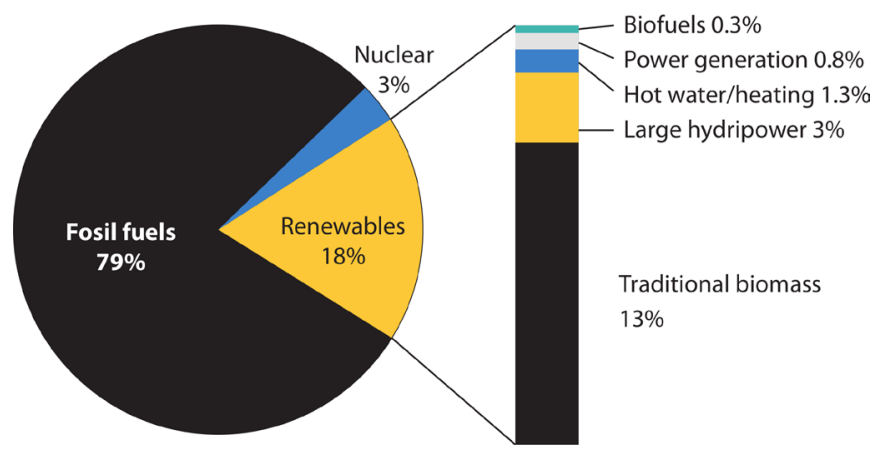

Figura 1. Contribución de la energía renovable respecto a energía primaria mundial.

El aprovechamiento de la energía solar para el suministro de energía eléctrica mediante un sistema fotovoltaico, SFV, está constituido básicamente por un generador FV y un equipo de acondicionamiento electrónico, que es diferente, dependiendo si el SFV opera en forma autónoma (SFVA) o interconectadamente (SFVI), El generador FV está constituido a su vez por un arreglo de módulos FV interconectados en serie y/o paralelo, dependiendo del tipo de carga para la cual se suministra el fluido eléctrico.

Los SFVA usan bancos de baterías de respaldo, ya que operan aisladamente de la red eléctrica y son instalados generalmente en zonas remotas y son usados para suministrar fluido eléctrico a comunidades rurales. Los elementos que conforman un sistema SFVA típico para estas regiones son: el generador fotovoltaico, banco de baterías, controlador de carga e inversor, que se usa cuando es necesario suministrar potencia eléctrica a carga AC. 


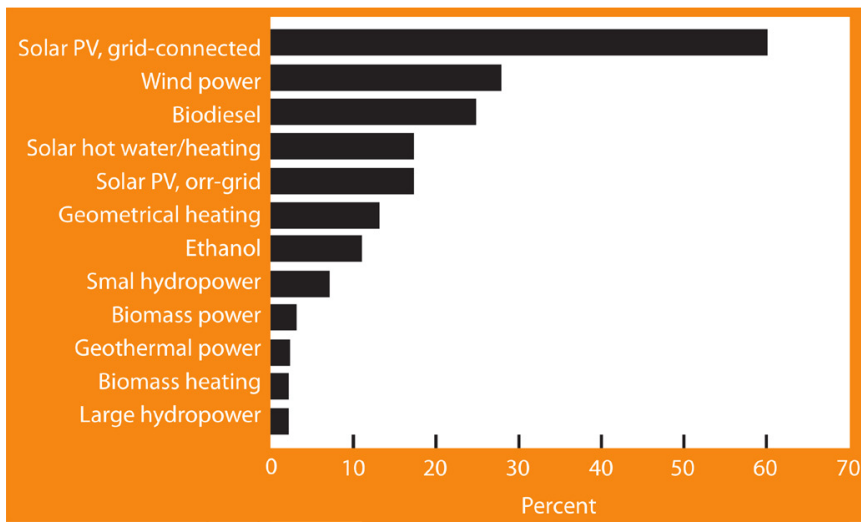

Figura 2. Crecimiento de las energías renovables a nivel mundial de 2002-2006.

En los países industrializados, gracias a los avances alcanzados en las tecnologías de dispositivos fotovoltaicos y convertidores estáticos de potencia, así como a la reducción de los costos de fabricación de módulos FV, la generación fotovoltaica se ha venido convirtiendo gradualmente en una alternativa viable en el esquema de generación distribuida. En él, una combinación de plantas centrales y un gran número de pequeños generadores dispersos en la red eléctrica satisfacen la demanda de electricidad. Este concepto es actualmente una realidad en algunos países como Holanda, Alemania, Dinamarca y Japón [1].

En Colombia se ha venido usando la energía solar fotovoltaica, generada básicamente a través de SFVA diseñada para suministrar potencia eléctrica a hogares y centros de salud en zonas remotas aisladas y para uso en telecomunicaciones. Según reportes obtenidos hasta el año 1998 [4], en Colombia habían instalados 60.000 SFVA con una potencia promedio de $60 \mathrm{Wp}$.

\section{Fundamentación teórica de la generación fotovoltaica}

Para convertir la energía del sol en energía eléctrica, se utilizan dispositivos semiconductores, llamados celdas solares, que convierten la radiación solar directamente en energía eléctrica, a través del fenómeno físico conocido como efecto fotovoltaico, que fue descubierto por Edmund Becquerel en 1839.

Estos dispositivos se fabrican fundamentalmente con dos tipos de estructura diferentes. En el primer tipo de estructura denominada homojuntura, los materiales semiconductores p y n que constituyen el dispositivo son iguales. Las celdas solares basadas en tecnología de silicio se fabrican con este tipo de estructura. El segundo tipo tiene una estructura denominada heterojuntura, debido a que en este caso los materiales semiconductores p y n que constituyen el dispositivo son diferentes. 
La celda solar más utilizada es la unión p/n cuyo funcionamiento en la oscuridad difiere muy poco del que presenta un diodo de unión y por consiguiente las características de tensión obedecen a los resultados típicos del diodo [5]. Bajo condiciones de iluminación se crean pares electrón-hueco en exceso sobre las concentraciones de equilibrio en el volumen del semiconductor, que son separadas por el campo eléctrico de la unión $\mathrm{p} / \mathrm{n}$ provocando la aparición de una corriente eléctrica en la carga conectada exteriormente.

En la figura 3 se muestra el circuito equivalente de una celda solar real. Este está constituido por un diodo que representa la juntura $\mathrm{p} / \mathrm{n}$ de la celda en oscuro Id, el cual está en paralelo con una fuente que representa la corriente generada por la celda bajo iluminación $I_{p h}$, una resistencia en serie $R_{s}$, que representa la resistencia óhmica a través de los materiales semiconductores, de los contactos eléctricos y de la rejilla metálica, y una resistencia en paralelo $R_{p}$ a través de la cual se presentan fugas de corriente.

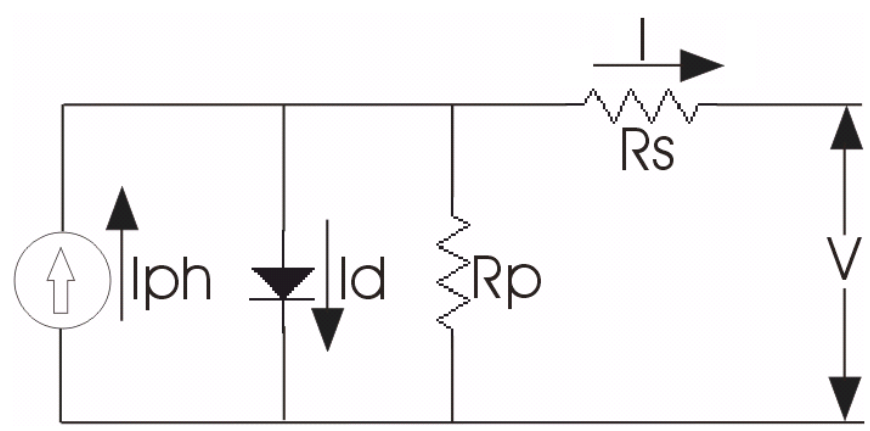

Figura 3. Circuito equivalente de una celda solar real.

El comportamiento de una celda o módulo solar, se representa en la siguiente ecuación.

$$
I=I_{p h}-I_{0}\left[\exp \left(\frac{q\left(V+I R_{s}\right)}{m k T}\right)-1\right]-\frac{V+I R_{s}}{R_{p}}
$$

Donde $I_{0}$ es la corriente de saturación del diodo, $m$ el factor de idealidad del diodo, $k$ la constante de Boltzmann $\left(k=1.38 \times 10^{-23} \mathrm{~J} / \mathrm{K}\right), T$ es la temperatura absoluta de la celda en K, y $q$ es la carga del electrón $\left(q=1.6 \times 10^{-19}\right.$ Coulomb). En las celdas que componen los módulos fotovoltaicos comerciales, la eficiencia es grande, por lo que la resistencia en paralelo es muy grande en relación con el numerador, el factor de idealidad del diodo para celdas de silicio es $m=1$, y además el término $k T / q$ se conoce como voltaje termodinámico $V_{T}$ quedando la ecuación (1) de la siguiente forma: 


$$
I=I_{p h}-I_{0}\left[\exp \left(\frac{V+I R_{s}}{V_{T}}\right)-1\right]
$$

\subsection{Caracterización de una celda solar}

El desempeño de una celda o módulo solar se determina generalmente a través de medidas de corriente, en función del voltaje de polarización (característica $\mathrm{I}-\mathrm{V})$. La figura 6 muestra la característica I-V típica de una celda solar en oscuro y bajo iluminación. La celda solar está constituida por un diodo que representa la juntura $\mathrm{p} / \mathrm{n}$ de la celda en oscuro, $I_{d}$, que está en paralelo con una fuente de corriente que representa la fotocorriente generada por la celda bajo iluminación $I_{p h}$. Idealmente, en una celda solar $R_{s}=0$ y $m=1$ es el factor de idealidad del diodo.

De la curva de I-V bajo iluminación, es posible determinar los parámetros que caracterizan el desempeño de un celda solar: corriente de corto circuito $\left(I_{S C}\right)$, voltaje de circuito abierto $\left(V_{O C}\right)$, máxima potencia $\left(P_{M}\right)$ y el factor de llenado $(F F)$.

La corriente de corto circuito $\left(I_{S C}\right)$ la corriente generada por la celda cuando el voltaje de polarización es cero. Esta es igual a la corriente fotogenerada por la celda y es directamente proporcional a la intensidad de la radiación solar incidente; la $I_{S C}$ no es afectada significativamente por la temperatura ambiente $[5]$.

El voltaje de circuito abierto $\left(V_{O C}\right)$ es la diferencia de potencial generada por la celda, cuando la corriente total en ésta es cero. La máxima potencia $\left(P_{M}\right)$ que produce la celda solar es igual a la potencia obtenida en el punto de máxima potencia "MPP" de la curva I-V. Si $I_{M}$ y $V_{M}$ son la corriente y voltaje generados por la celda en el MPP, entonces la potencia máxima $P_{M}$ que puede ser generada por la celda es igual al producto $I_{M} \times V_{M}$.

\subsection{Dimensionado de un sistema fotovoltaico autónomo}

Para dimensionar un SFVA existen diversos criterios [6,7]. Sin embargo, para lograr un dimensionamiento óptimo es necesario tener datos confiables de radiación solar y temperatura ambiente del lugar donde se instala el sistema y hacer un balance confiable de la potencia que puede ser generada por el SFV y la potencia consumida por la carga [6]. Adicionalmente, se requiere tener información de la radiación solar del mes de menos radiación del año, que se expresa en HPS (horas promedio de radiación solar estándar diaria: $1000 \mathrm{~W} / \mathrm{m}^{2}$ ).

Como existen épocas del año con bajo nivel de radiación solar la demanda tiende a superar la capacidad de generación del SFV, es indispensable diseñar un perfil de consumo diario del usuario para evitar una suspensión de la energía eléctrica. Este perfil se diseña teniendo en cuenta un uso racional del consumo, de tal forma que solo se gaste energía eléctrica para satisfacer las necesidades más prioritarias del usuario. El perfil incluye básicamente el número de horas 
que deben diariamente estar encendidos los equipos que constituyen la carga del sistema, para que el consumo no exceda a la potencia generada por el SFV.

El cálculo del consumo de energía $E_{i}$ de cada elemento de carga se realiza con base en el conocimiento de la potencia nominal de cada aparato y el número de horas que este demora diariamente en operación, y en el sistema internacional de unidades se mide en Wh.

$$
E_{i}=P_{i} t_{i}
$$

La energía $E_{d}$ que se consume por día se calcula teniendo en cuenta los aparatos que trabajan en tensión directa (DC) y los que necesitan tensión (AC).

$$
E_{d}=\sum E_{i D C}+\sum \frac{E_{i A C}}{\eta}
$$

La carga de consumo diario $C_{d}$ medida en Amperios-hora se obtiene dividiendo la energía $\left(E_{d}\right)$ sobre la tensión nominal del banco de baterías $V_{B}$.

$$
C_{d}=\frac{E_{d}}{V_{B}}
$$

Como algunos electrodomésticos funcionan con tensión alterna, se hace necesario incluir en el SFV un dispositivo que convierta la tensión DC del banco de baterías en tensión AC.

El cálculo de la capacidad $C_{B}$ del banco de baterías se realiza teniendo en cuenta el consumo diario de carga $C_{d}$, el número de días que la batería puede suministrar la energía necesaria para la vivienda en ausencia de radiación (días de autonomía $d_{a}$ ) y la profundidad de descarga de la batería $P_{d}$, que es suministrada por el fabricante.

$$
C_{B}=\frac{C_{d} d_{a}}{P_{d}}
$$

El cálculo de la potencia que el generador FV debe producir se efectúa teniendo en cuenta que ésta debe ser mayor que la energía consumida en la carga. Por consiguiente, es necesario conocer en forma confiable la radiación solar promedio que incide en el lugar de instalación. Adicionalmente, es conveniente dimensionar el generador $\mathrm{FV}$, teniendo en cuenta un factor de seguridad $F_{S}$ de 1.2 para compensar las pérdidas por efectos de temperatura, caída de tensión en los cables, cubrimiento de los módulos con polvo, inclinación de los módulos y otros factores.

La potencia $P_{G}$ producida por el generador $\mathrm{FV}$, se puede calcular mediante la siguiente relación:

$$
P_{G}=\frac{E_{d}}{(H P S) F_{S}}
$$

Por consiguiente, la energía eléctrica $E_{G}$ producida por el generador FV es:

$$
E_{G}=P_{G}(H P S)
$$

Donde HPS es el número de horas promedio de radiación solar estándar diaria. 
El número de módulos en paralelo $N_{p}$ del generador $\mathrm{FV}$ se determina a través de la siguiente relación:

$$
N_{p}=\frac{C_{G}}{I_{M}(H P S)}
$$

donde $I_{M}$ es la corriente pico producida por el módulo $\mathrm{FV}$ seleccionado y $C_{G}$ es la carga que el generador debe suministrar al banco de baterías, que está dada por:

$$
C_{G}=\left(\frac{E_{G}}{V_{M G}}\right)
$$

El número de módulos en serie del generador FV se determina teniendo en cuenta la tensión del generador $V_{G}$ y la tensión nominal de los módulos a través de la siguiente relación:

$$
N_{s}=\left(\frac{V_{G}}{V_{N}}\right)
$$

El número total de módulos $N_{T}$ está dado por:

$$
N_{T}=N_{s} N_{p}
$$

Para evitar las sobre cargas y descargas profundas del banco de baterías que disminuyen la vida útil de las mismas, se hace necesario instalar un controlador de carga para que las características del mismo se determinen, teniendo en cuenta la corriente máxima que produce el generador fotovoltaico; un valor aproximado se puede obtener de acuerdo con el número de módulos conectados en paralelo $N_{p}$ y la corriente de máxima potencia $I_{M}$ de cada módulo, así:

$$
I_{R}=N_{p} I_{M}
$$

La potencia del inversor se selecciona teniendo en cuenta la potencia máxima de consumo en tensión alterna $P_{A C}$ y la eficiencia de conversión del inversor $\eta$, por lo que la potencia $P_{i n v}$ que debe suministrar el inversor es:

$$
P_{i n v}=\frac{P_{A C}}{\eta}
$$

Para dimensionar adecuadamente un SFV es necesario conocer los parámetros eléctricos de salida del módulo FV seleccionado, la radiación solar y temperatura ambiente promedio en el sitio de instalación del SFV y las características de la carga (potencia consumida, tensión de los equipos que conforman la carga y tiempo de consumo). Los parámetros eléctricos del módulo son suministrados por el fabricante, y son medidos bajo condiciones de radiación y temperaturas estándar usando un simulador solar.

\section{Materiales y métodos}

El proyecto fue desarrollado en una vivienda rural ubicada en la vereda de ceiba pareja perteneciente al corregimiento de Nariño en la margen izquierda 
del Rio Sinú en el municipio de Lorica en el Departamento de Córdoba. Ceiba pareja es una vereda localizada a unos $10 \mathrm{~km}$ de la cabecera municipal y donde habitan unas 40 familias que derivan su sustento de la agricultura y la ganadería, pero con vías de acceso muy deterioradas por el paso del invierno y la falta de mantenimiento.

\subsection{Diseño de estudio}

Para el dimensionamiento se consultó el perfil de usuario de la planta solar de acuerdo con la información obtenida de los habitantes de la vivienda, esta información se encuentra en el cuadro 1. La planta fue instalada el 4 de Enero de 2009 y se evaluó su comportamiento y desempeño hasta el 14 de Mayo de 2010. Se escogió el sitio porque hasta el año 2007 esta vereda no contaba con el servicio de interconexión a red y esto era determinante en la cultura del uso racional de energía. Otro aspecto importante era que se necesitaba que en el sitio existiera conexión a la red convencional para alimentar el sistema de monitoreo.

Tipo de electrodoméstico Cantidad Tiempo de uso por día (h)

\begin{tabular}{cll}
\hline Televisor & 1 & 6 \\
\hline Radio & 1 & 5 \\
\hline Ventiladores & 2 & 8 \\
\hline Lámparas & 5 & 4 \\
\hline Otros & 1 & 2 \\
\hline
\end{tabular}

Cuadro 1. Perfil de usuario de la planta solar. Fuente: información obtenida en entrevista con los habitantes de la vivienda.

\subsection{Análisis estadístico}

El programa desarrollado permitió medir el desempeño del sistema, lo que permitió medir las variables que inciden en el desempeño de la planta solar, como son: la radiación solar global, temperatura del aire sobre los módulos fotovoltaicos, la potencia suministrada por los módulos fotovoltaicos y la potencia de consumo de la vivienda. Estas variables fueron medidas automáticamente minuto a minuto entre 12:05 am hasta las 11:55 pm de cada día con algunas interferencias debido a fallas en el fluido eléctrico que alimentaba al sistema de monitoreo.

Con los valores medidos se hicieron promedios diarios anuales de radiación solar global, temperatura ambiente, potencia generada por la planta solar y energía suministrada por la misma. Adicionalmente se determinaron los valores promedios durante un día claro de la eficiencia de conversión de los módulos y la eficiencia del inversor. 


\subsection{Sistema de monitoreo para evaluar el desempeño del sistema}

En sistema de monitoreo desarrollado permitió monitorear en tiempo real las siguientes variables que determinan el desempeño de la planta solar: potencia del generador, nivel de tensión del banco de baterías, consumo de energía DC y AC y con esto se determina la eficiencia del generador FV. Para obtener la información de las variables físicas involucradas se usaron los siguientes sensores y transductores [8].

- Radiación: Fotodiodo de Silicio Kipp \& Zonen SP-LITE, Tiempo de respuesta: $<1 \mathrm{seg}$, sensibilidad nominal: $72 \mathrm{mV} / \mathrm{W} / \mathrm{m}^{2}$, Rango espectral: 0,4-1,1 $\mu \mathrm{m}$.

- Temperatura ambiente: Un termistor NTC de $10 \mathrm{k} \Omega$ conectado en serie con una resistencia $R=5,01 \mathrm{k} \Omega$ y alimentado por una fuente regulada de $5 \mathrm{~V}$ de la tarjeta NI USB 6008.

- Tensión DC: Divisor de tensión con relación (5/1).

- Corriente DC: Pinza FLUKE i410, con las siguientes características: Señal de salida: $1 \mathrm{mV}$ por Amperio de AC ó DC; Tensión de trabajo: 600 Vrms.; Impedancia de carga: >1 MW, <100 pF; Gama de corriente utilizable: 0, 5400A.

\section{Resultados del dimensionado de los sistemas fotovoltaicos}

Para tener en cuenta los efectos que produce la temperatura sobre los módulos, se desarrolló un programa que permite calcular las características eléctricas de módulos FV de Si-Mono o Policristalino bajo cualquier condición de radiación y temperatura a partir de los datos que suministra el fabricante y los resultados obtenidos de la estación instalada en el municipio de Lorica.

\subsection{Dimensionado del sistema fotovoltaico autónomo}

Para el dimensionado del sistema, se desarrolló un programa en el lenguaje de programación gráfica en LabVIEW 8.5, que calcula las características de este tipo de sistemas y que soluciona numéricamente la ecuación (2), con la información obtenida de los datos que reporta el fabricante de módulos fotovoltaicos Tenesol, el perfil de usuario que se reporta en el cuadro 1 y las medidas realizadas tanto de radiación como temperatura en el sitio de instalación [1], se realizó el dimensionamiento de la planta solar. En el cuadro 2 se muestran las características eléctricas de los principales componentes del sistema dimensionado e instalado. En la figura 4 se presenta el diagrama del sistema fotovoltaico instalado en la vereda de Ceiba Pareja en el municipio de Lorica.

\subsection{Resultados del monitoreo del sistema fotovoltaico}

En la figura 5 se puede observar los valores de radiación y temperatura alcanzados en días con atmósfera despejada; además se indican valores de radiación con 


\begin{tabular}{ccc}
\hline Elemento & Cantidad & Descripción \\
\hline Módulos fotovoltaicos & 4 & $145 \mathrm{Watt} / 12 \mathrm{VDC}$ \\
\hline Controlador de carga & 2 & $20 \mathrm{~A} / 12 \mathrm{VDC}$ \\
\hline Inversor & 1 & $400 \mathrm{Watt} / \mathrm{VDC}$ \\
\hline Banco de baterías & 2 & $250 \mathrm{Ah} / \mathrm{VDC}$ \\
\hline
\end{tabular}

Cuadro 2. Resultado del dimensionado.

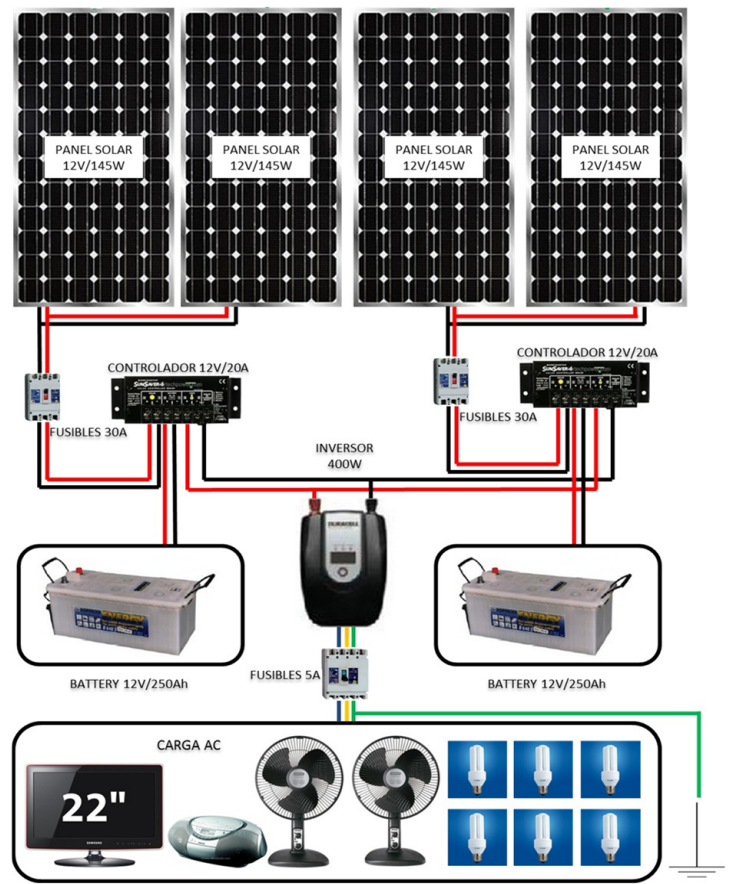

Figura 4. Esquema del sistema fotovoltaico autónomo instalado en la vereda Ceiba Pareja.

valores cercanos a los 1000 Watt $/ \mathrm{m}^{2}$ en las horas del mediodía, lo que evidencia la dispersión de la radiación solar en la atmósfera debido a que no se alcanzan valores del orden de $1300 \mathrm{Watt} / \mathrm{m}^{2}$ presentes cuando hay movimiento de nubes que permiten la entrada de radiación solar directa. En relación con la temperatura, se observa una relación similar con valores de temperatura más elevada en las horas de la tarde, lo que hace que la potencia de los módulos se vea un poco disminuida por este efecto. 
En la figura 6 se aprecia cómo la tensión del módulo se ve muy poco afectada por la radiación, en cambio la corriente del generador guarda una proporcionalidad con la radiación solar, lo que es entendible debido a que un módulo fotovoltaico es una fuente de corriente.

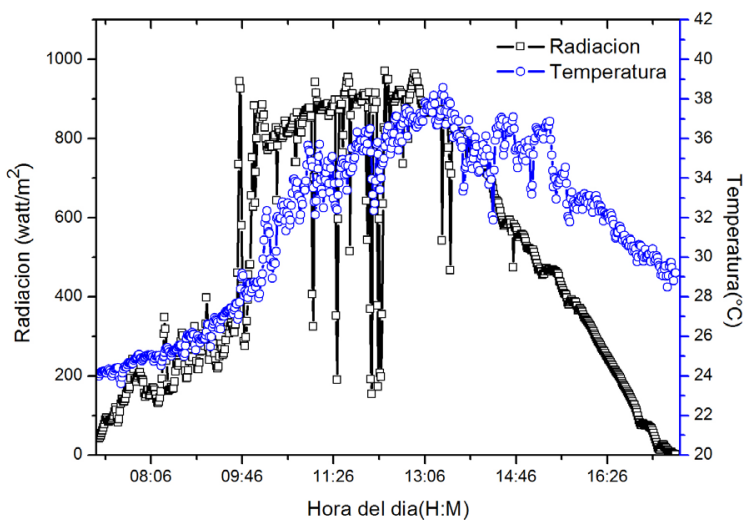

Figura 5. Se muestran los valores de radiación solar y temperatura ambiente.

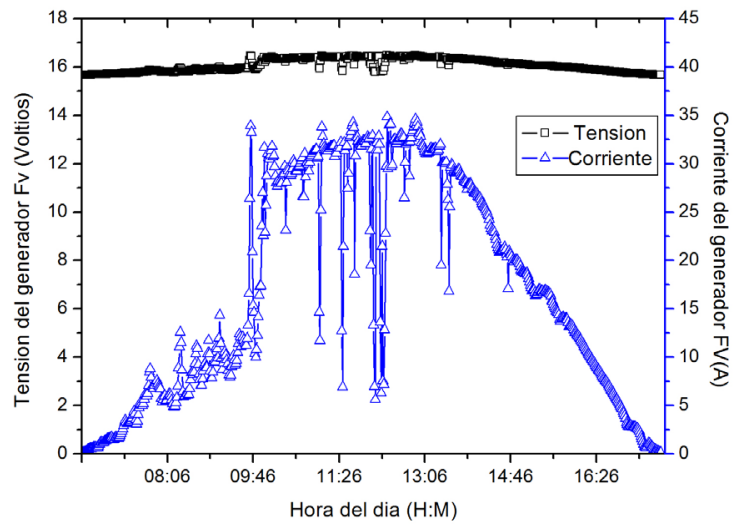

Figura 6. Tensión y corriente en generador fotovoltaico. 
En las figuras 7 y 8 se observa cómo la potencia de la radiación solar es mayor que la potencia generada por el módulo fotovoltaico, y esto obedece a las pérdidas que existen en el módulo, en especial, por calentamiento, mecanismos de recombinación en la celda, perdidas en la interconexión de celdas y otros fenómenos físicos dentro de la celda.

Para este caso calculamos la eficiencia de conversión del generador usando la ecuación (14), y se observa sus variaciones entre el $11 \%$ y el $12 \%$ y la eficiencia que reportan los fabricantes de los módulos fotovoltaicos son del orden del $13 \%$.

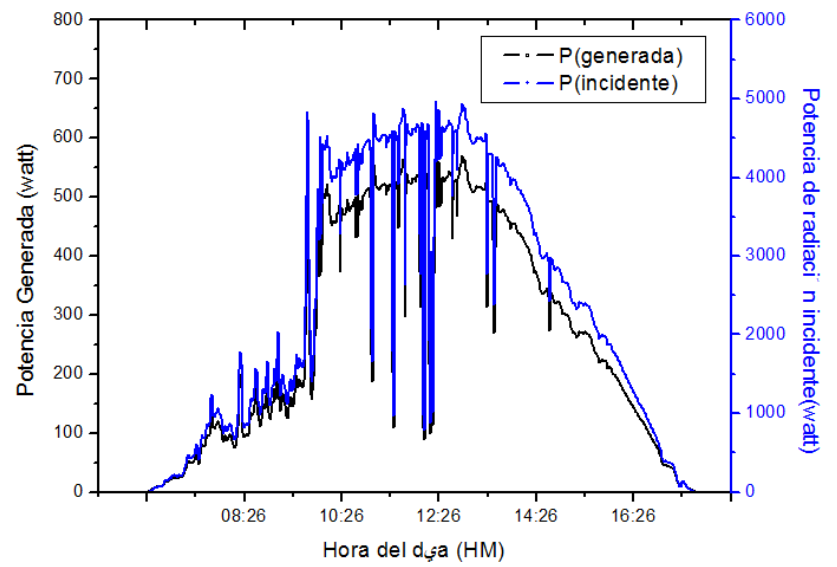

Figura 7. Se muestran los valores de potencia generada por el conjunto de módulos y la potencia contenida en la radiación incidente.

En cuanto a la eficiencia del inversor, se pudo verificar que usando la ecuación (13), haciendo medidas de potencia de entrada (DC) y salida (AC) con todos los electrodomésticos conectados y operando para el inversor Xantrex 400 Watt, la eficiencia varía entre un $85 \%$ y $95 \%$ siendo menor cuando se tienen operando menores electrodomésticos, es decir, la eficiencia del inversor depende de la potencia en la que opera siendo mayor cuando más cerca se está de la potencia nominal.

\subsection{Resultados promedios mensuales}

Los valores de energía en (Wh) y la radiación acumulada promedio (Watt $/ \mathrm{m}^{2}$ ) fueron promediados y los resultados se muestran en la figura 9. La energía solar incidente medida sobre la superficie de módulos fotovoltaicos, muestra que los meses de mayor generación de energía corresponden a los meses de mayores valores de radiación solar acumulada con valores máximos de generación de 3000 


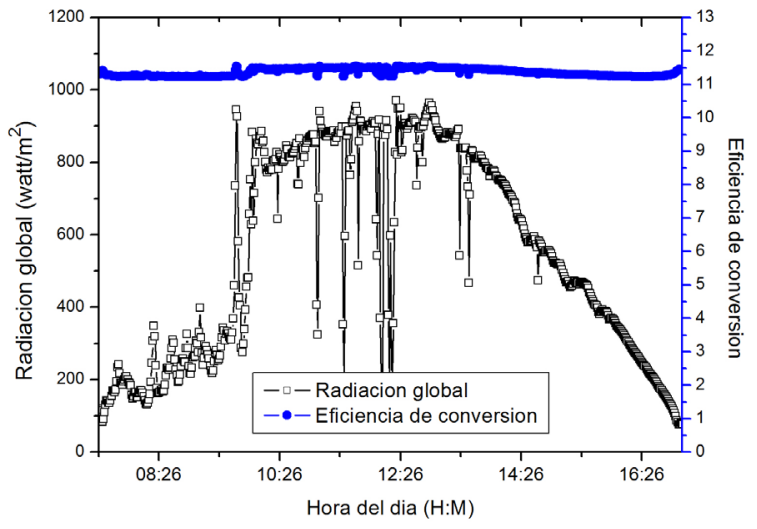

Figura 8. La radiación global y la eficiencia de conversión del generador fotovoltaico.

Wh en el mes de febrero y los valores mínimos de generación correspondientes a los meses de abril y mayo que es la temporada de mayor pluviosidad y baja radiación en el municipio de Lorica, con promedio generado de $2700 \mathrm{Wh}$, lo que generó las tres fallas presentadas en el sistema durante el año de operación.

La variación promedio de la tensión en el banco de baterías se agudiza en los meses de más baja radiación, esto se ve compensado por que en estos meses se ve disminuido el consumo de energía debido a una disminución en las horas de uso de los ventiladores y esta situación se refleja en la figura 10 .

\section{Análisis de los resultados}

Se observa que al presentarse una temperatura más elevada en las horas de la tarde, la potencia de los módulos disminuye, este efecto obedece a las pérdidas que existen en el módulo, en especial, por calentamiento del material semiconductor que permite la reducción de la tensión, además de otros fenómenos físicos dentro de la celda, que hacen que la eficiencia de conversión sea del orden del 12,5\%.

La eficiencia del inversor varía entre $85 \%$ y $95 \%$ siendo menor cuando se tienen operando menores electrodomésticos, esto indica que se debe utilizar un inversor con potencia nominal un $10 \%$ arriba de la potencia máxima de carga AC conectada simultáneamente.

La energía solar incidente medida sobre la superficie de módulos fotovoltaicos, muestra que los meses de mayor generación de energía corresponden a los meses de mayores valores de radiación solar acumulada con valores máximos de generación de 3000 Wh en el mes de febrero; y los valores mínimos de generación correspondientes a los meses de abril y mayo en el municipio de Lorica la energía 


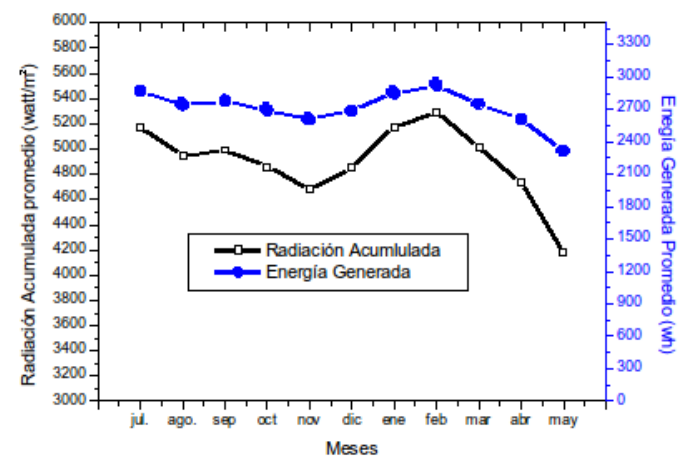

Figura 9. Radiación acumulada promedio y energía generada diaria.

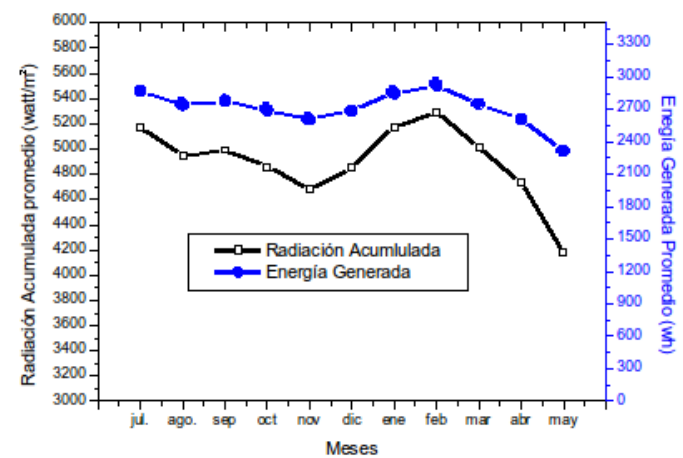

Figura 10. Energía generada o producida por la planta solar.

generada fue la más baja (2700 Wh) y fue donde se presentaron las tres fallas de la planta durante el año de operación.

\section{Conclusiones}

Los resultados del dimensionamiento de la planta para esta vivienda, usando el programa DISIFVA, sugirieron la instalación de un generador fotovoltaico de 580 Watts y un banco de baterías de dos días de autonomía que forma un banco de baterías de $500 \mathrm{Ah} / 12 \mathrm{VDC}$. Los resultados del monitoreo de la planta solar evidenciaron los siguientes resultados: 
- La estabilidad de la tensión y corriente AC, obtenidas mediante el uso de inversores de onda sinusoidal, en contraparte con la inestabilidad en tensión y corriente predominantes en las redes de interconexión de la empresa Electricaribe en la misma zona.

- La confiabilidad de una planta solar autónoma, cuando se dimensiona teniendo en cuenta no sólo la radiación solar promedio del sitio sino la temperatura promedio; estriba cuando se hace uso racional de la energía. La planta solar presentó tres fallas durante el año de operación, mientras que la red de interconexión de la empresa Electricaribe, para la misma zona y el mismo periodo presentó veinte y seis fallas.

- Las fallas presentadas en la planta solar fueron ocasionadas en parte porque los habitantes de la vivienda usaron más de la energía definida en el perfil de usuario y también debido que a en los meses de abril y mayo fue donde se presentó más baja la radiación. Para suplir esta deficiencia se sugirió hacer un incremento en la capacidad del banco de baterías de 500Ah a 800Ah.

- La eficiencia de conversión de los módulos fotovoltaicos usados en este proyecto es de $12,3 \%$ para temperaturas del orden de $30{ }^{\circ} \mathrm{C}$ y se ve disminuido $11,4 \%$ en las horas del mediodía cuando la temperatura ambiente alcanza valores entre los $35{ }^{\circ} \mathrm{C}$ y $40{ }^{\circ} \mathrm{C}$. Se dimensionó un sistema fotovoltaico para suministrar energía eléctrica a una vivienda localizada en la vereda de Ceiba Pareja en la zona rural del municipio de Lorica. Para esta vivienda se diseñó un sistema fotovoltaico con una potencia de $580 \mathrm{Wp}$.

- La viabilidad y recuperación de la inversión inicial se tiene cuando la distancia de la vivienda a la red de interconexión es mayor a $1 \mathrm{~km}$. El costo que debe asumir el propietario de la vivienda rural por kilómetro de instalación es del orden $\$ 10$ ’000.000, lo que garantiza que la instalación de energía solar fotovoltaica es una excelente alternativa para los habitantes de la zona rural del municipio de Lorica.

\section{Referencias}

1. Narváez, J.R., Gordillo, G., Dielman, G.: Estudio del Potencial de Radiación Solar y de la Temperatura Ambiente en Lorica Córdoba, RCF vol. 40, No. 2, Julio (2008)

2. Renewable 2005,: Global Status Report. Eric Martinot, REN 21 Renewable Energy Policy Networks 2005. Washington, DC: Worldwatch Institute. (2005)

3. Diniz, A.S., Rosa, J.G.F., Almarenga, C.A.: Proc. $2^{\text {nd }}$ World Conference on Solar Energy Conversion, Wien (1998)

4. Rodríguez, H., González, F.: Portafolio Colombiano de Proyectos para MDL, Academia Colombiana de Ciencias, Bogotá, agosto (2000)

5. Herrera, O., Quiñones, C., Gordillo, G., Medida y simulación teórica de la característica I-V de una Celda solar, Revista Colombiana de Física, vol. 33, No. 2. (2001)

6. Rodríguez, M.: Energías Renovables, Editorial Thompson - Paraninfo, (1999)

7. Delima, R.: Diseño de sistema fotovoltaico para vivienda rural unifamiliar, Fundación para el Desarrollo de la Ciencia y la Tecnología, Venezuela, (2000)

8. Hernandez-Mora, J., Gordillo, G.: Diseño e instalación de primer sistema fotovoltaico interconectado en Colombia y desarrollo de equipo para su monitoreo, Tesis de maestría en Ingeniería Universidad Nacional de Colombia (2006) 